\title{
Applications of Nonstandard Finite Difference Methods to Nonlinear Heat Transfer Problems
}

\author{
Alaeddin Malek \\ Department of Applied Mathematics, Faculty of Mathematical Sciences, Tarbiat Modares \\ University, P.O. Box 14115-134, Tehran,
}

Iran

\section{Introduction}

This chapter presents some applications of nonstandard finite difference methods to general nonlinear heat transfer problems. Nonlinearity in heat transfer problems arises when

i. Some properties in the problem are temperature dependent

ii. Boundary conditions are described by nonlinear functions

iii. The interface energy equation in phase change problems is nonlinear.

Free convection and surface radiation are famous examples of nonlinear boundary conditions. The temperature distribution in multi phase regions is governed by various heat equations, for example in two phase region the temperature is governed by two different heat equation, one for solid phase, and the other one for liquid phase. Conservation of energy at a phase change interface usually yields to the nonlinear boundary condition.

While particular problems presented in this research relates to nonlinear heat transfer in a thin finite rod, I fell that the methodology by which one solves these problems by nonstandard finite difference methods are quite general. I hope that these bits and pieces will be taken as both a response to a specific problem and a general method.

It is common to model the nonlinear heat transfer problems by parabolic time dependent nonlinear partial differential equations. The model assumes a certain separation between time and space which is present in parabolic heat transfer problems. There are two approaches for the solution of nonlinear parabolic PDEs. One is the closed analytical form solution based on the separation of variables. Although the method of separation of variables has wide applicability, it is most often limited to linear problems. The second approach is numerical approach based on the discretization of the region. There are various approaches for performing the discretization, such as finite difference (M. Necati Ozisik et al., 1994; D. R. Croft \& D. G. Lilly, 1977), finite element (R. W. Lewis, et al., 2005; J. M. Bergheau, 2010), finite volume (S. V. Patankar, 1980; H. K. Versteeg \& W. Malalasekera, 1996), and spectral methods (G. Ben-Yu, 1998; O. P. Le Maitre \& O. M. Knio, 2010). Here we concentrate on both standard and nonstandard finite difference methods. Standard FDs are usually used for linear part of the problem while the nonstandard FDs are used to deal with nonlinearity. Since corresponding PDEs in the heat transformation are some time dependent equations, in general one need to consider both discretizations in time and in the space. There are two typical discretization techniques in the literature. First is the semi- 
discertization scheme and the second is fully discertization formulation. In semidiscertization, we treat the time variable $t$ as a specific variable among the independent variables, i.e., for a fixed $t$ we discretize the spatial domain and derivatives first. This usually yields to a system of initial value ordinary differential equations. After this stage any of the ODE methods and software may be used to solve the problem. Some of the well known methods in this context are method of lines, Euler and Runge-Kutta methods. An obvious process to obtain a full discretization scheme for the time dependent PDEs such as parabolic equations is to discretize in time the corresponding semi-discretization scheme.

Nonlinear partial differential equations are defined as the mathematical representation of real world problems concerned with the determination of a solution of a function of several variables, which are required to satisfy a number of initial and boundary conditions. Such function computations are sought in diverse fields, including heat transform, fluid mechanics, electrical and industrial engineering, medical sciences and denoising a noisy image.

Although many classical numerical algorithms such as finite difference, finite element, control volume, boundary element and spectral methods are given to solve linear and nonlinear heat transfer problems, in many applications, it is easier and desire to have nonstandard FD discretization for the corresponding nonlinear terms. However, traditional standard finite difference algorithms are not suitable for nonlinear heat transfer problems. In the present work we will show that the nonstandard finite difference approach is one of the promising approaches that can handle the nonlinearity and perform an accurate numerical solution to the problem.

In the recent years many nonstandard FD models developed to solve nonlinear heat transfer problems. Several basic and advance questions associated with these models have motivated the studies presented in this chapter.

The goal of this chapter is threefold. First we consider the nonlinear conduction problems and discuss the sources of nonlinearity in these problems. Second, the theoretical areas of interest include fundamental methods, models and algorithms for solving general nonlinear heat transfer problems using various implicit and explicit nonstandard finite difference methods is discussed. Third, we will try to present and discuss the numerical analysis for the corresponding models, simulations and applications of nonstandard methods that solve various practical heat transfer problems.

Nonstandard finite difference methods are an area of finite difference methods which is one of the fundamental topics of the subject that coup with the nonlinearity of the problem very well. This subject combines many mathematical concepts like ordinary and partial differential equations, nonlinear boundary conditions, consistency, convergence, stability and error analysis of the corresponding algorithms. Students are often familiar with the steady and time dependent ODEs with one variable before embarking on an undergraduate course, and in practical way will have function derivatives which they cannot express in numerical form. Here we must compute the solution for the nonlinear time dependent PDEs with one dependent and of several independent variables. From mathematical point of the view convergence of the solution, consistency and stability of the method has quiet importance, while as an engineer one might look for an efficient algorithm that works for many different real life problems.

The numerical analysis and mathematical techniques considered here are some service industry in order to write some reliable codes to solve nonlinear heat transfer problems. The 
theoretical analysis forms a tool to determine bounds on various kinds of errors and levels of stability. These error bounds form the basis of a theoretical justification for the solution convergence of the corresponding numerical algorithm to the actual solution of the original nonlinear heat transfer model. Whichever numerical algorithm is used, one should take care of the consistency between the nonstandard FD model and the original heat transfer problem.

The chapter covers a range of topics from undergraduate work on standard finite difference solution to ODEs and PDEs through to recent research on nonstandard finite difference schemes for nonlinear heat transfer problems. The source of the standard finite difference work is the lecture notes for graduate students participated in my advance course in the numerical solution to PDEs. The notes have grown in nineteen years of teaching the subject. The work on nonstandard finite difference models is based partly on my own research. Part of this research started in the year 2000 during the time that I was in university of Pretoria, South Africa in the sabbatical leave from Tarbiat Modares University, Tehran, Iran. It has taken annual updates as new models have proposed in some of the thesis of my postgraduate students during the last six years. This research is enriched by the literature published with Mickens and his co-author during the last fifteen years.

I am grateful to the applied mathematics department in the faculty of mathematical sciences at Tarbiat Modares University which has made available the technical equipment and environment for the research. The novel models and numerical programs have been tested, compared and improved using the different computers which have been installed over the years.

In the next section we study general concepts introductory in the heat transfer problems. Then, under the assumption that there exists a solution we try to seek an efficient numerical solution using nonstandard FD methods.

\section{Heat transfer preliminaries}

\section{Definition 2.1:}

In general, the process by which molecules spread from areas of high concentration, to areas of low concentration is called diffusion.

In the following we give some ideas and examples representing heat convection, conduction and radiation. These facts or events in the real world as they are experienced by the human senses are three physical concepts that heat transfer problems engage one or some combinations of them.

\subsection{Conduction}

Conduction is the transfer of energy through matter from particle to particle. It is a heat transfer directly through materials, occurring when mass of material stays in place. It is the transfer and distribution of heat energy from atom to atom within a substance. For example, a spoon in a cup of hot soup becomes warmer because the heat from the soup is conducted along the spoon. Conduction is most effective in solids but it can happen in fluids.

Have you ever noticed that metals tend to feel cold? They are not colder. They only feel colder because they conduct heat away from your hand. The human being recognizes the heat that is leaving his hand as cold. This makes it clear that: what identifies heat is temperature difference and not temperature. 
Conduction heat transfer phenomenon is encountered in many real life problems. One may consider the following few examples among the broad range of conduction problems.

- Electronic Devices Design: An electronic package is to be cooled by conduction. A heat sink consisting of small devices is recommended to maintain the electronic components below a specified temperature. How many devices of what size and material and configuration are necessitated?

- Liquid in Glass Thermometer: Liquids like mercury and alcohol are used to measure temperature by the technique of putting the liquid in glass. Determine the minimum and maximum temperature that mercury and alcohol can measure by this technique of thermometer manufacturing.

- Heat Loss of an Aluminum Alloy Plate: An aluminum alloy plate is suddenly quenched into liquid oxygen. Find the heat loss by the plate. Calculate the required time for the plate to reach to specific temperature.

- Nuclear Reactor Coolant Pump: The temperature of some nuclear elements begins to rise, if the coolant pump fails. Determine the required time that engineers need to fix the pump before meltdown happens.

- $\quad$ Space Vehicle Reentry Safeguard: A heat guard is used to protect a space vehicle during reentry. The shield ablates as it passes through the atmosphere. Specify the required safeguard thickness and material to protect a space vehicle during reentry.

- Supersonic Rocket Nozzle Protection: The throat of a supersonic rocket nozzle is protected by inserting a porous ring at the throat. Injection of helium through the ring lowers the temperature and protects the nozzle. Determine the amount of helium needed to protect a rocket nozzle during a specified trajectory.

\subsubsection{Classical heat conduction theory (linear)}

In the case of classical heat conduction it has been demonstrated many times that if two parallel plates of the same area $A$ and different constant temperatures $T_{1}$ and $T_{2}$ respectively, are separated by a small distance $d$, an amount of heat per unit time will pass from the warmer to cooler. This amount of heat is proportional to the area $A$, the temperature difference $\left|T_{1}-T_{2}\right|$, and inversely proportional to the separation distance $d$. Thus

$$
\text { amount of heat per unit time }=\kappa A \frac{\left|T_{1}-T_{2}\right|}{d},
$$

where the positive factor $\kappa$ the material conductivity depends only on the material between the two plates. This is a physical law called Newton's low of cooling and has often been verified by careful experiment. Although, it is an experimental theory, it is the basis of the mathematical theory of classical heat conduction. This is a reason that in the classical theory of diffusion, Fourier's equation relates the heat flux to temperature according to the equation:

$$
q(r, \tau)=-\kappa \nabla T(r, \tau),
$$

where $q(r, \tau)$ is the heat flux, $\tau$ stands for time, $r$ is a position vector that has heat flux components in the $x, y$ and $z$ directions and $\kappa$ is the material conductivity. In this theory the medium is of macroscale and it is assumed that the heat flux vector and temperature gradient across a material volume occur at the same instant of time. 
The simple linear diffusion problem in one space variable $x$ and time $\tau$, for $(x, \tau) \in(0, l) \times(0, \infty)$, is (J. D. Smith, 1985)

$$
\frac{\partial T}{\partial \tau}=\kappa \frac{\partial^{2} T}{\partial X^{2}}
$$

The non-dimensionalizing process is illustrated below with the parabolic heat conduction equation (2.2).

\section{Work Example 1: (Involves only heat conduction)}

The solution of Eq. (2.2) gives the temperature $T$ at a distance $X$ from one end of a thin uniform wire after a time $\tau$. This assumes the rod is ideally heat insulated along its length and heat transfers at its ends. Let $l$ represent the length of the wire and $T_{0}$ some particular non negative constant temperature such as the maximum or minimum temperature at zero time.

Using the following dimensionless variables

$$
u=T / T_{0}, \quad x=X / l, \quad t=\kappa \tau / l^{2},
$$

equation (2.2) with the general boundary condition and specific initial temperature distribution, can be rewritten in the following dimensionless form

$$
\left\{\begin{array}{l}
u_{t}=u_{x x}, \quad(x, t) \in(0,1) \times(0, \infty) \\
u(0, t)=U_{1}, \quad u(1, t)=U_{2}, \quad t>0 \\
u(x, 0)=2 x, \quad x \in[0,1 / 2] \\
u(x, 0)=2(1-x), \quad x \in[0,1 / 2]
\end{array}\right.
$$

where $U_{1}$ and $U_{2}$ are the dimensionless forms of $T_{1}$ and $T_{2}$, respectively.

In other word we are seeking a numerical solution of $\frac{\partial u}{\partial t}=\frac{\partial^{2} u}{\partial x^{2}}$ which satisfies

\section{Case I:}

i. $\quad u=0$ at $x=0$ and $u=0$ at $x=l$ for all $t>0$.

ii. for $t=0: u=2 x$ for $0 \leq x \leq 1 / 2$ and $u=2(1-x)$ for $1 / 2 \leq x \leq 1$,

\section{Case II:}

iii. $\quad u=0$ at $x=0$ and $u=0$ at $x=l$ for all $t>0$.

iv. for $t=0: u=\sin \pi x$ for $0 \leq x \leq 1$.

where (i), (iii) and (ii), (iv) are called the boundary condition and the initial condition respectively.

\subsection{Convection}

Convection is the transfer of heat by the actual movement of the warmed matter. It is a heat transfer through moving fluid, where the fluid carries the heat from the source to destination. For example heat leaves the coffee cup as the currents of steam and air rise. Convection is the transfer of heat energy in a gas or liquid by movement of currents. It can 
also happen in some solids, like sand. More clearly, convection is effective in gas and fluids but it can happen in solids too. The heat current moves with the gas and fluid in the most of the food cooking. Convection is responsible for making macaroni rise and fall in a pot of heated water. The warmer portions of the water are less dense and therefore, they rise. Meanwhile, the cooler portions of the water fall because they are denser.

While heat convection and conduction require a medium to transfer energy, heat radiation does not. The energy travels through nothingness (vacuum) in the heat radiation.

\subsection{Radiation}

Electromagnetic waves that directly transport energy through space is called radiation. Heat radiation transmits by electromagnetic waves that travel best in a vacuum. It is a heat transfer due to emission and absorption of electromagnetic waves. It usually happens within the infrared/visible/ultraviolet portion of the spectrum. Some examples are: heating elements on top of toaster, incandescent filament heats glass bulb and sun heats earth. Sunlight is a form of radiation that is radiated through space to our planet without the aid of fluids or solids. The sun transfers heat through 93 million miles of space. There are no solids like a huge spoon touching the sun and our planet. Thus conduction is not responsible for bringing heat to Earth. Since there are no fluids like air and water in space, convection is not responsible for transferring the heat. Therefore, radiation brings heat to our planet.

Heat excites the black surface of the vanes more than it heats the white surface. Black is a good absorber and a good radiator. Think of black as a large doorway that allows heat to pass through easily. In contrast, white is a poor absorber and a poor radiator of energy. White is like a small doorway and will not allow heat to pass easily.

Note that heat transfer problems involve temperature distribution not just temperature. Heat transfer rates are determined knowing the temperature distribution. While Fourier's law of conduction provides the rate of heat transfer related to heat distribution, temperature distribution in a medium governs with the principle of conservation of energy.

\subsubsection{Stefan-Boltzmann radiation law}

If a solid with an absolute surface temperature of $T$ is surrounded by a gas at temperature $T_{\infty}$, then heat transfer between the surface of the solid and the surrounding medium will take place primarily by means of thermal radiation if $\left|T-T_{\infty}\right|$ is sufficiently large (P. M. Jordan, 2003). Mathematically, the rate of heat transfer across the solid-gas interface is given by the Stefan-Boltzmann radiation law

$$
\kappa(\partial T / \partial n)_{s}=-\sigma \varepsilon A\left(T^{4}-T_{\infty}^{4}\right),
$$

where $(\partial T / \partial n)_{s}$ the thermal gradient at the surface of the solid is evaluated in the direction of the outward-pointing normal to the surface, $A$ is radiating area and $\kappa>0$ is the thermal conductivity of the solid (assumed constant). The constants $\varepsilon \in[0,1],(\varepsilon=1$ for ideal radiator while for a prefect insulator $\varepsilon=0)$ and $\sigma \approx 5.67 \times 10^{-8} \mathrm{~W} /\left(\mathrm{m}^{2} \mathrm{~K}^{4}\right)$ are, respectively, the emissivity of the surface and the Stefan-Boltzmann constant (P. M. Jordan, 2003). Mathematically, the rate of heat transfer across the solid-gas interface is given by the Newton's law of cooling (H. S. Carslaw \& J. C. Jaeger, 1959; R. Siegel \& J. H. Howell, 1972)

$$
\kappa(\partial T / \partial n)_{s}=-h A\left(T-T_{\infty}\right),
$$


where $h$ is the convection heat transfer coefficient and $A$ is cooloing area.

The applications of thermal radiation with/without conduction can be observed in a good number of science and engineering fields including aerospace engineering/design, power generation, glass manufacturing and astrophysics (R. Siegel \& J. H. Howell, 1972; L. C. Burmeister, 1993; M. N. Ozisik, 1989; J. C. Jaeger, 1950; E. Battaner, 1996).

In the following Work Examples we consider two problems that involve various heat transfer properties in a thin finite rod (A. Mohammadi \& A. Malek, 2009).

\section{Nonlinear heat transfer in a finite thin wire}

\subsection{Heat transfer involving both conduction and radiation}

In the following example we consider a problem that involves both conduction and radiation and no convection.

Consider a very thin, homogeneous, thermally conducting solid rod of constant crosssectional area $A$, perimeter $p$, length $l$ and constant thermal diffusivity $\kappa>0$ that occupies the open interval $(0, l)$ along the $X$ - axis of a Cartesian coordinate system. That $T$ the temperature distribution of the $\operatorname{rod}$, is $T(X, \tau)$, and $T_{0} \sin (\pi X / l)$ is initial temperature of the rod, and let the ends at $X=0, l$ be maintained at the constant temperatures $T_{1}$ and $T_{2}$ respectively and $T_{\infty}$ the surrounding temperature. The parabolic one-dimensional unsteady heat conduction model in a thin finite rod that is radiating heat across its lateral surface into a medium of constant temperature is the mathematical model of this physical system consists of the following initial boundary value problem (P. M. Jordan, 2003; W. Dai \& S. Su, 2004)

$$
\left\{\begin{array}{l}
T_{\tau}=\kappa T_{X X}-\beta_{0}\left(T^{4}-T_{\infty}^{4}\right), \quad(X, \tau) \in(0, l) \times(0, \infty) \\
T(0, \tau)=T_{1}, \quad T(l, \tau)=T_{2}, \quad \tau>0 \\
T(X, 0)=T_{0} \sin (\pi X / l), \quad X \in(0, l)
\end{array}\right.
$$

where time $\tau$ is a non-negative variable, $\beta_{0}=\kappa \sigma \varepsilon p / K A$ in wich $K$ is relative thermal diffusivity constant and $A$ stands for radiation area, and based on physical considerations, $T$ is assumed to be nonnegative.

\section{Work Example 2: (Involves heat conduction and heat radiation)}

Using the following dimensionless variables

$$
\begin{aligned}
& u=T / T_{0}, \quad x=X / l, \quad t=\kappa \tau / l^{2}, \\
& \beta=T_{0}^{3} l^{2} \sigma \varepsilon p / K A, \quad u_{\infty}=T_{\infty} / T_{0},
\end{aligned}
$$

where $T_{0}>0$ is taken as constant, problem (3.1) can be rewritten in dimensionless form as follows (P. M. Jordan, 2003; W. Dai \& S. Su, 2004):

$$
\left\{\begin{array}{l}
u_{t}=u_{x x}-\beta\left(u^{4}-u_{\infty}^{4}\right), \quad(x, t) \in(0,1) \times(0, \infty) ; \\
u(0, t)=U_{1}, \quad u(1, t)=U_{2}, \quad t>0 \\
u(x, 0)=\sin \pi x, \quad x \in(0,1)
\end{array}\right.
$$

where $U_{1}$ and $U_{2}$ are the dimensionless forms of $T_{1}$ and $T_{2}$, respectively. 


\subsection{Heat transfer in a finite thin rod with additional convection term}

Problem (3.1) with additional convection term becomes:

$$
\left\{\begin{array}{l}
T_{\tau}=\kappa T_{X X}-\beta_{0}\left(T^{4}-T_{\infty}^{4}\right)-\alpha_{0}\left(T-T_{\infty}\right),(X, \tau) \in(0, l) \times(0, \infty) ; \\
T(0, \tau)=T_{1}, \quad T(l, \tau)=T_{2}, \quad \tau>0 \\
T(X, 0)=T_{0} \sin (\pi X / l), \quad X \in(0, l)
\end{array}\right.
$$

where $\tau$ the temporal is a non-negative variable, $\beta_{0}=\kappa \sigma \varepsilon p / K A, \alpha_{0}=\kappa h p / K A$, and based on physical considerations, $T$ is assumed to be nonnegative.

\section{Work Example 3: (Involve conduction, radiation and convection terms)}

Using the following dimensionless variables,

$$
\begin{gathered}
u=T / T_{0}, \quad x=X / l, \quad t=\kappa \tau / l^{2}, \quad \beta=T_{0}^{3} l^{2} \sigma \varepsilon p / K A, \\
\alpha=l^{2} h p / K A, \quad u_{\infty}=T_{\infty} / T_{0},
\end{gathered}
$$

where $T_{0}>0$ is taken as constant, problem (3.4) can be rewritten in dimensionless form as follows:

$$
\left\{\begin{array}{l}
u_{t}=u_{x x}-\beta\left(u^{4}-u_{\infty}^{4}\right)-\alpha\left(u-u_{\infty}\right),(x, t) \in(0,1) \times(0, \infty) \\
u(0, t)=U_{1}, \quad u(1, t)=U_{2}, \quad t>0 \\
u(x, 0)=\sin \pi x, \quad x \in(0,1)
\end{array}\right.
$$

where $U_{1}$ and $U_{2}$ are the dimensionless forms of $T_{1}$ and $T_{2}$, respectively.

In the following we propose six nonstandard explicit and implicit schemes for problem (3.6).

\section{Novel heat theory (Microscale)}

Tzou (D. Y. Tzou, 1997) has shown that if the scale in one direction is at the microscale (of order 0.1 micrometer) then the heat flux and temperature gradient occur in this direction at different times. Thus the heat conduction equations used to describe the microstructure thermodynamic behavior are:

$$
-\nabla . q+Q=\rho c_{p} \frac{\partial T}{\partial \tau} \text { and } Q\left(r, \tau+\tau_{Q}\right)=-\kappa \nabla T\left(r, \tau+\tau_{T}\right),
$$

where $\rho, c_{p}$ and $Q$ are density, a specific heat and a heat source, $\tau_{Q}$ and $\tau_{T}$ are the time lags of the heat flux and temperature gradient which are positive constants.

Now we can introduce (A. Malek \& S. H. Momeni-Masuleh, 2008) the novel heat equation as:

$$
\begin{aligned}
\frac{\rho c_{p}}{\kappa}\left(\frac{\partial T}{\partial \tau}+\tau_{q} \frac{\partial^{2} T}{\partial \tau^{2}}\right) & =\nabla^{2} T+\tau_{q}\left(\frac{\partial^{3} T}{\partial \tau \partial x^{2}}+\frac{\partial^{3} T}{\partial \tau \partial y^{2}}\right)+\tau_{T} \frac{\partial^{3} T}{\partial \tau \partial z^{2}}+ \\
& \frac{\left(Q+\tau_{q} \frac{\partial Q}{\partial \tau}\right)}{\kappa}
\end{aligned}
$$

Malek and Momeni-Masuleh in years 2007 and 2008 used various hybrid spectral-FD methods to solve Eq. (3.7) efficiently. H. Heidari and A. Malek, studied null boundary controllability for hyperdiffusion equation in year 2009. Heidari, H. Zwart, and Malek, in year 2010 discussed 
controllability and stability of the 3D novel heat conduction equation in a submicroscale thin film. In this Chapter we consider the heat theory for macroscale objects. Thus we do not consider the numerical solution for Eq. (3.7) that is out of the scope of this chapter.

\section{Finite difference methods}

\subsection{Standard finite difference methods}

In this section, we shall first consider two well known standard finite difference methods and their general discretization forms. Second, we shall introduce semi-discretization and fully discretization formulas. Third we will consider consistency, convergence and stability of the schemes. We will consider the nonlinear heat transfer problems in the next section during the study of nonstandard FD methods. This, as we shall see, leads to discovering some efficient algorithms that exists for corresponding class of nonlinear heat transfer problems.

Among the class of standard finite difference schemes, two important and richly studied subclasses are explicit and implicit approaches.

\section{Notation}

It is useful to introduce the following difference notation for the first derivative of a function $u$ in the $x$ direction at discrete point $j$ throughout this Chapter.

$$
\begin{aligned}
& \left(\frac{\partial u}{\partial x}\right)_{j}=\frac{u_{j+1}-u_{j}}{\Delta x} \quad \text { Forward Finite Difference } \\
& \left(\frac{\partial u}{\partial x}\right)_{j}=\frac{u_{j}-u_{j-1}}{\Delta x} \quad \text { Backward Finite Difference } \\
& \left(\frac{\partial u}{\partial x}\right)_{j}=\frac{u_{j+1 / 2}-u_{j-1 / 2}}{\Delta x} \quad \text { Central Finite Difference }
\end{aligned}
$$

The equation $\frac{\partial u}{\partial t}=\frac{\partial^{2} u}{\partial x^{2}}$ may be approximated at the point $(i \Delta x, j \Delta t)$ by the difference equation:

$$
\frac{u_{i, j+1}-u_{i, j}}{\Delta t}=\frac{\theta\left(u_{i+1, j+1}-2 u_{i, j+1}+u_{i-1, j+1}\right)+(1-\theta)\left(u_{i+1, j}-2 u_{i, j}+u_{i-1, j}\right)}{(\Delta x)^{2}},
$$

for $0 \leq \theta \leq 1$, where $u_{i, j}=u(i \Delta x, j \Delta t)$ for $i=1, \ldots N$ and $j=1, \ldots, J$ in the $x-t$ plane. Note that $\theta=0$ gives the explicit scheme and $\theta=1 / 2$ represents the Crank-Nicolson method that is one of the famous implicit FD schemes.

\subsubsection{Explicit standard FD scheme $(\theta=0)$}

We calculate an explicit standard finite difference solution of the problem given in Work Example 1 for both Cases I and II, where the closed analytical form solutions are

$$
U=\frac{8}{\pi^{2}} \sum_{n=0}^{\infty} \frac{1}{n^{2}}\left(\sin \frac{1}{2} n \pi\right)(\sin n \pi x) e^{-n^{2} \pi^{2} t} \text { and } U=e^{-\pi^{2} t} \sin \pi x \text { respectively. }
$$

Figs. 1, 2, 3 and 4 display the power of both numerical schemes (Explicit and CrankNicolson) for the calculation of the solution for problems given in Work Example 1. 


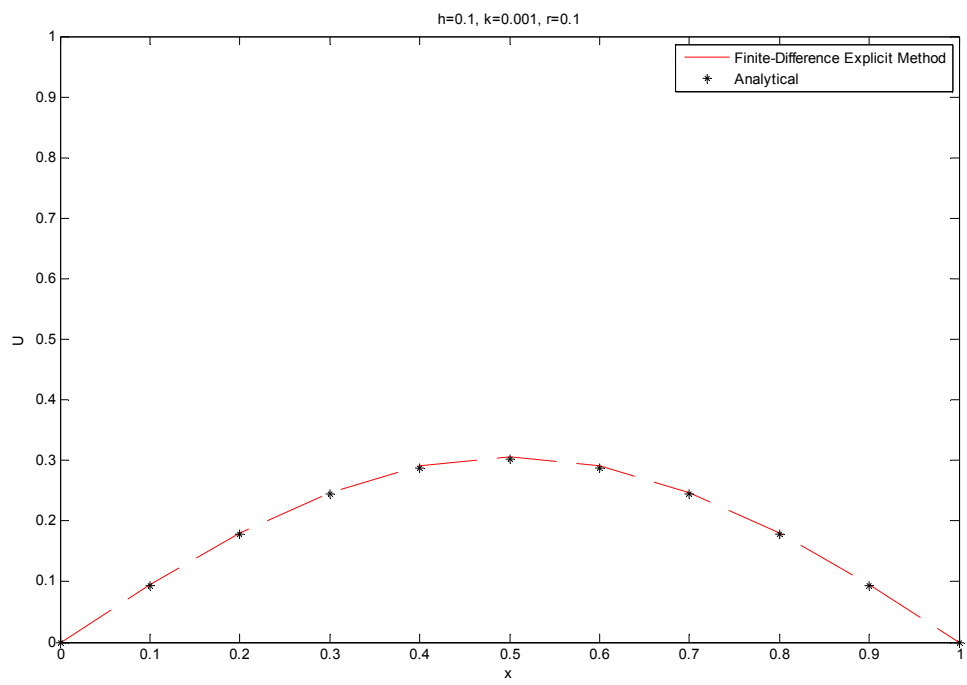

Fig. 1. Standard explicit FD solution of Work Example 1, Case I.

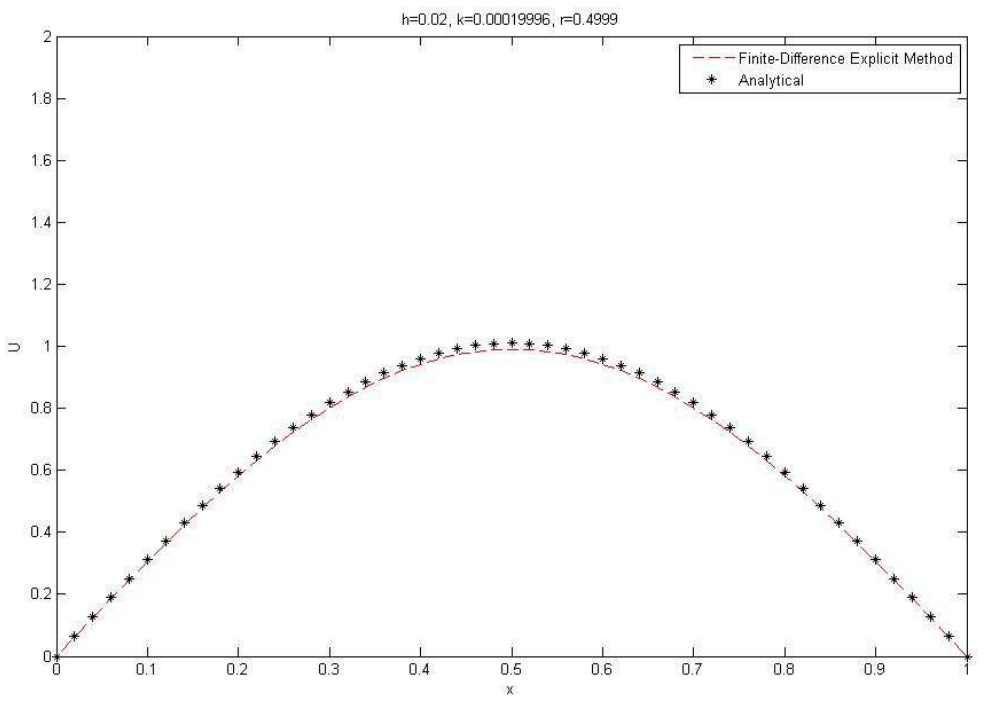

Fig. 2. Standard explicit FD solution of Work Example 1, Case II.

\subsubsection{Crank-Nicolson standard FD scheme ( $\theta=1 / 2)$}

We calculate a Crank-Nicolson implicit solution of the problem given in Work Example 1 for Case I and Case II. 


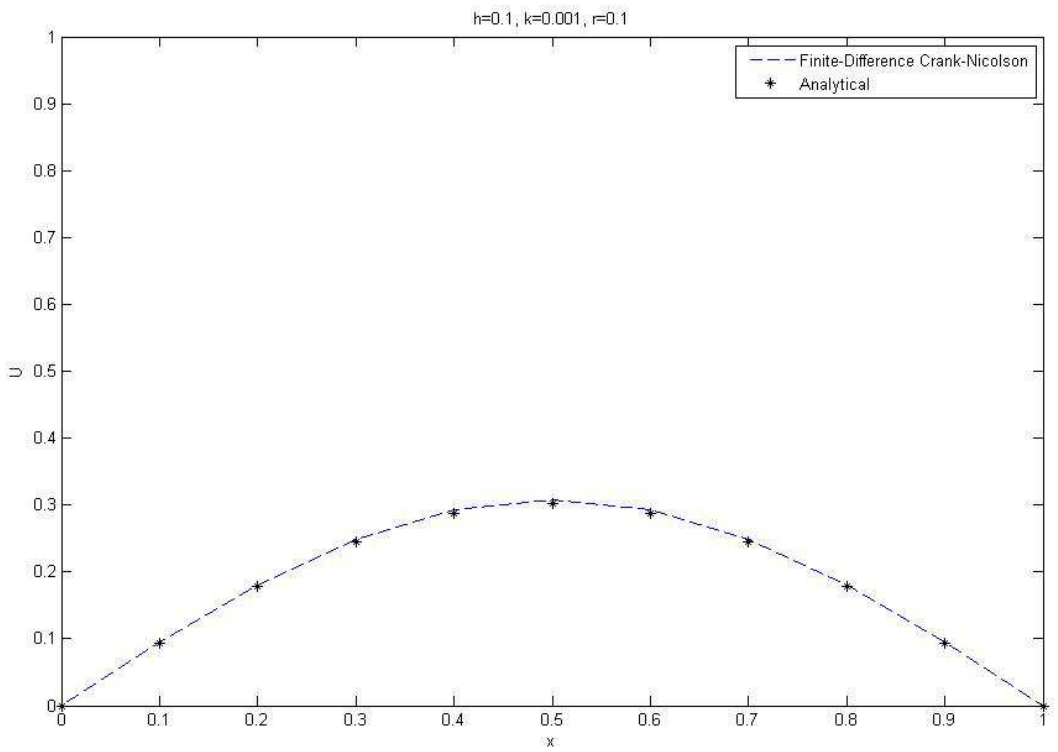

Fig. 3. Crank-Nicolson implicit FD solution for Work Example 1, Case I.

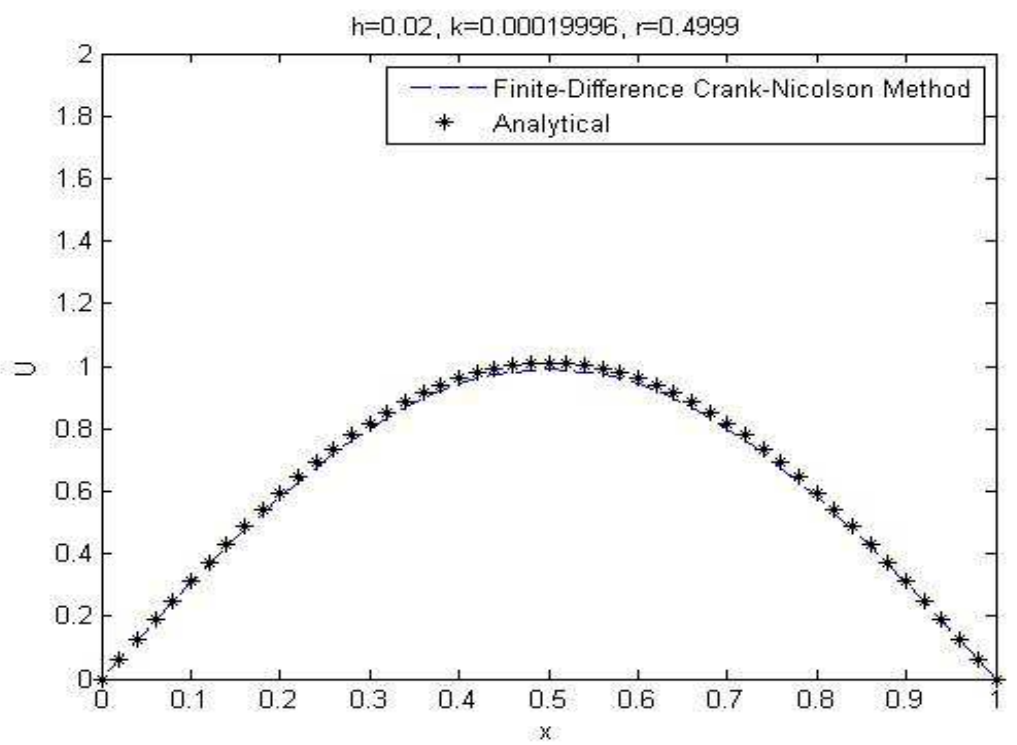

Fig. 4. Crank-Nicolson implicit FD solution of Work Example 1, Case II.

Up to this point most of our discussion has dealt with standard finite difference methods for solving differential equations. We have considered linear equations for which there is a well-designed and extensive theory. Some simple diffusion problems without nonlinear 
terms were considered in Section 4.1. Now we must face the fact that it is usually very difficult, if not impossible, to find a solution of a given differential equation in a reasonably suitable and unambiguous form, especially if it involves the nonlinear terms. Therefore, it is important to consider what qualitative information can be obtained about the solutions of differential equation, particularly nonlinear terms, without actually solving the equations.

\subsection{Nonstandard finite difference methods}

Nonstandard finite difference methods for the numerical integration of nonlinear differential equations have been constructed for a wide range of nonlinear dynamical systems (P. M. Jordan, 2003; W. Dai \& S. Su, 2004; H. S. Carslaw \& J. C. Jaeger, 1959; R. Siegel \& J. H. Howell, 1972; L. C. Burmeister, 1993). The basic rules and regulations to construct such schemes (R. E. Mickens, 1994), are:

Regulation 1. To do not face numerical instabilities, the orders of the discrete derivatives should be equal to the orders of the corresponding derivatives appearing in the differential equations.

Regulation 2. Discrete representations for derivatives must have nontrivial denominator functions.

Regulation 3. Nonlinear terms should be replaced by nonlocal discrete representations.

Regulation 4. Any particular properties that hold for the differential equation should also hold for the nonstandard finite difference scheme, otherwise numerical instability will happen.

Positivity, boundedness, existence of special solutions and monotonicity are some properties of particular importance in many engineering problems that usually model with differential equations. Regulation number four restricts one to force the nonstandard scheme satisfying properties of differential equation.

In the last two decays, several nonstandard finite difference schemes have been developed for solving nonlinear partial differential equations by Mickens and his co-authors. Particularly, Jordan and Dai considered a problem of one-dimensional unsteady heat conduction in a thin finite rod that is radiating heat across its lateral surface into a medium of constant temperature. The most fundamental modes of heat transfer are conduction and thermal radiation. In the former, physical contact is required for heat flow to occur and the heat flux is given by Fourier's heat law. In the latter, a body may lose or gain heat without the need of a transport medium, the transfer of heat taking place by means of electromagnetic waves or photons.

In the reminder of this Chapter, we consider twelve nonstandard implicit and explicit difference schemes for nonlinear heat transfer problems involving conductions and radiation with or without convection term. Specifically, we employ the highly successful nonstandard finite difference methods (A. Mohammadi, \& A. Malek, 2009) to solve the nonlinear initial-boundary value problems in Work Examples 2 and 3 (see Section 3). We show that the third implicit schemes are unconditionally stable for large value of the equation parameters with or without convection term. It is observed that the rod reaches steady state sooner when it is exposed both to the radiation heat and convection.

\subsection{Explicit nonstandard FD schemes}

\subsubsection{Nonstandard FD explicit schemes for Work Example 2}

In Ref. (P. M. Jordan, 2003; W. Dai \& S. Su, 2004), three nonstandard explicit finite difference schemes for Eq. (3.3) are developed as follows: 


$$
\begin{aligned}
& u_{i, j+1}=\frac{u_{i, j}(1-2 r)+r\left(u_{i-1, j}+u_{i+1, j}\right)+\beta(\Delta t) u_{\infty}^{4}}{1+\beta(\Delta t)\left(u_{i, j}^{3}\right)}, \\
& u_{i, j+1}=\frac{u_{i, j}(1-2 r)+r\left(u_{i-1, j}+u_{i+1, j}\right)+\beta(\Delta t) u_{\infty}^{4}}{1+\beta(\Delta t)\left(u_{i-1, j}^{3}+u_{i+1, j}^{3}\right) / 2},
\end{aligned}
$$

and

$$
u_{i, j+1}=\frac{u_{i, j}(1-2 r)+r\left(u_{i-1, j}+u_{i+1, j}\right)+\beta(\Delta t)\left(u_{i, j}^{2}+u_{\infty}^{2}\right)\left(u_{i, j}+u_{\infty}\right) u_{\infty}}{1+\beta(\Delta t)\left(u_{i, j}^{2}+u_{\infty}^{2}\right)\left(u_{i, j}+u_{\infty}\right)},
$$

where $r \equiv \Delta t /(\Delta x)^{2}$, and $u_{i, j}=u(i \Delta x, j \Delta t), \Delta x$ is the grid size and $\Delta t$ is the time increment. While these three schemes differ in the way of dealing with the nonlinear terms, truncation errors for all of them are of the order $O\left[\Delta t+(\Delta x)^{2}\right]$.

Equation (4.3) has better stability property than Eq. (4.1) and (4.2), ( for more details see A. Mohammadi, \& A. Malek, 2009). This scheme satisfies the positivity condition, i.e., we can conclude that if $u_{i, j}>0 \Rightarrow u_{i, j+1}>0$, whenever $r \leq \frac{1}{2}$. Moreover this scheme is stable for large values of the equation parameters comparing with the nonstandard schemes (4.1) and (4.2).

\subsubsection{Nonstandard FD explicit schemes for Work Example 3}

Three nonstandard explicit finite difference schemes are introduced (A. Mohammadi, \& A. Malek, 2009) with additional convection heat transfer phenomenon as follows:

$$
\begin{aligned}
& u_{i, j+1}=\frac{u_{i, j}(1-2 r)+r\left(u_{i-1, j}+u_{i+1, j}\right)+\beta(\Delta t) u_{\infty}^{4}+\alpha(\Delta t) u_{\infty}}{\left(1+\beta(\Delta t)\left(u_{i, j}^{3}\right)+\alpha(\Delta t)\right)}, \\
& u_{i, j+1}=\frac{u_{i, j}(1-2 r)+r\left(u_{i-1, j}+u_{i+1, j}\right)+\beta(\Delta t) u_{\infty}^{4}+\alpha(\Delta t) u_{\infty}}{\left(1+\left(\beta(\Delta t)\left(u_{i-1, j}^{3}+u_{i+1, j}^{3}\right) / 2\right)+\alpha(\Delta t)\right)},
\end{aligned}
$$

and

$$
\frac{u_{i, j}(1-2 r)+r\left(u_{i-1, j}+u_{i+1, j}\right)+\beta(\Delta t)\left(u_{i, j}^{2}+u_{\infty}^{2}\right)\left(u_{i, j}+u_{\infty}\right) u_{\infty}+\alpha(\Delta t) u_{\infty}}{\left(1+\beta(\Delta t)\left(u_{i, j}^{2}+u_{\infty}^{2}\right)\left(u_{i, j}+u_{\infty}\right)+\alpha(\Delta t)\right)} .
$$

\subsection{Implicit nonstandard FD schemes}

\subsubsection{Nonstandard FD implicit schemes for Work Example 2}

Finite differencing methods can be employed to solve the system of equations and determine approximate temperatures at discrete time intervals and nodal points. Problem (3.3) is solved numerically using the non-standard Crank-Nicholson method. To provide accuracy, difference approximations are developed at the midpoint of the time increment. 
A second derivative in space is evaluated by an average of two central difference equations, one evaluated at the present time increment $j$ and the other at the future time increment $j+1$ :

$$
\frac{\partial^{2} u}{\partial x^{2}}=\frac{1}{2}\left(\frac{u_{i-1, j+1}-2 u_{i, j+1}+u_{i+1, j+1}}{(\Delta x)^{2}}+\frac{u_{i-1, j}-2 u_{i, j}+u_{i+1, j}}{(\Delta x)^{2}}\right)
$$

where $j$ represents a temporal node and $i$ represents a spatial node.

Making these substitutions into Eq. (3.3), gives

$$
\frac{u_{i, j+1}-u_{i, j}}{\Delta t}=\frac{1}{2}\left(\frac{u_{i-1, j+1}-2 u_{i, j+1}+u_{i+1, j+1}}{(\Delta x)^{2}}+\frac{u_{i-1, j}-2 u_{i, j}+u_{i+1, j}}{(\Delta x)^{2}}\right)-\beta\left(u^{4}-u_{\infty}^{4}\right) .
$$

Now define

$$
\begin{aligned}
& u^{4} \rightarrow u_{i, j}^{3} u_{i, j+1}, \quad u_{i, j}^{3} \equiv\left(u_{i-1, j}^{3}+u_{i+1, j}^{3}\right) / 2, \\
& \quad \beta\left(u^{4}-u_{\infty}^{4}\right) \rightarrow \beta\left(u_{i, j}^{2}+u_{\infty}^{2}\right)\left(u_{i, j}+u_{\infty}\right)\left(u_{i, j+1}-u_{\infty}\right) .
\end{aligned}
$$

In this study, three nonstandard implicit finite difference schemes are developed as follows (A. Mohammadi, \& A. Malek, 2009)

$$
\begin{gathered}
-r u_{i-1, j+1}+\left(2+2 r+\beta(\Delta t) u_{i, j}^{3}\right) u_{i, j+1}-r u_{i+1, j+1}= \\
r u_{i-1, j}+(2-2 r) u_{i, j}+r u_{i+1, j}+\beta(\Delta t) u_{\infty}^{4}, \\
-r u_{i-1, j+1}+\left(2+2 r+\beta(\Delta t)\left(u_{i-1, j}^{3}+u_{i+1, j}^{3}\right) / 2\right) u_{i, j+1}-r u_{i+1, j+1}= \\
r u_{i-1, j}+(2-2 r) u_{i, j}+r u_{i+1, j}+\beta(\Delta t) u_{\infty}^{4},
\end{gathered}
$$

and

$$
\begin{aligned}
& -r u_{i-1, j+1}+\left(2+2 r+\beta(\Delta t)\left(u_{i, j}^{2}+u_{\infty}^{2}\right)\left(u_{i, j}+u_{\infty}\right)\right) u_{i, j+1}-r u_{i+1, j+1}= \\
& r u_{i-1, j}+\left(2-2 r+\beta(\Delta t) u_{\infty}\left(u_{i, j}^{2}+u_{\infty} u_{i, j}+u_{\infty}^{2}\right)\right) u_{i, j}+r u_{i+1, j}+\beta(\Delta t) u_{\infty}^{4}
\end{aligned}
$$

where $t \rightarrow t_{k}=(\Delta t) k, \quad x \rightarrow x_{m}=(\Delta x) m$. It can be seen that the truncation errors are of the order $O\left[(\Delta t)^{2}+(\Delta x)^{2}\right]$. In the Section 4.3, we prove that the scheme (4.12) is stable.

\subsubsection{Nonstandard FD implicit schemes for Work Example 3}

Three nonstandard implicit finite difference schemes are proposed (A. Mohammadi, \& A. Malek, 2009) with regard to convection heat transfer as follows:

$$
\begin{gathered}
-r u_{i-1, j+1}+\left(2+2 r+\beta(\Delta t) u_{i, j}^{3}+\alpha(\Delta t)\right) u_{i, j+1}-r u_{i+1, j+1}= \\
r u_{i-1, j}+(2-2 r) u_{i, j}+r u_{i+1, j}+\beta(\Delta t) u_{\infty}^{4}+\alpha(\Delta t) u_{\infty}, \\
-r u_{i-1, j+1}+\left(2+2 r+\left(\beta(\Delta t)\left(u_{i-1, j}^{3}+u_{i+1, j}^{3}\right) / 2\right)+\alpha(\Delta t)\right) u_{i, j+1}-r u_{i+1, j+1}= \\
r u_{i-1, j}+(2-2 r) u_{i, j}+r u_{i+1, j}+\beta(\Delta t) u_{\infty}^{4}+\alpha(\Delta t) u_{\infty},
\end{gathered}
$$


and

$$
\begin{aligned}
& -r u_{i-1, j+1}+\left(2+2 r+\beta(\Delta t)\left(u_{i, j}^{2}+u_{\infty}^{2}\right)\left(u_{i, j}+u_{\infty}\right)+\alpha(\Delta t)\right) u_{i, j+1}-r u_{i+1, j+1}= \\
& r u_{i-1, j}+\left(2-2 r+\beta(\Delta t) u_{\infty}\left(u_{i, j}^{2}+u_{\infty} u_{i, j}+u_{\infty}^{2}\right)\right) u_{i, j}+r u_{i+1, j}+\beta(\Delta t) u_{\infty}^{4}+\alpha(\Delta t) u_{\infty} .
\end{aligned}
$$

\subsection{Stability analysis for nonstandard FD implicit schems}

The questions considered in this section are mainly associated with the idea of stability of a solution. In the simplest form it makes it clear that: is whether small changes in the initial conditions (inputs) lead to small changes (stability) or to large changes (instability) in the computed solution (output).

Consider the stability of the nonstandard implicit finite difference scheme (4.12) where the coefficients are constant values. If the boundary values at $i=0$ and $N$, for $j>0$, are known, these $(N-1)$ equations for $i=1 \ldots N-1$ can be written in matrix form as

$$
\begin{aligned}
& {\left[\begin{array}{ccccccc}
M & -r & & & & & \\
-r & M & -r & & & & \\
& \cdot & \cdot & \cdot & & & \\
& & \cdot & \cdot & \cdot & & \\
& & & \cdot & \cdot & \cdot & \\
& & & & -r & M & -r \\
& & & & & -r & M
\end{array}\right]\left[\begin{array}{c}
u_{1, j+1} \\
u_{2, j+1} \\
\cdot \\
\cdot \\
\cdot \\
u_{N-2, j+1} \\
u_{N-1, j+1}
\end{array}\right]=}
\end{aligned}
$$

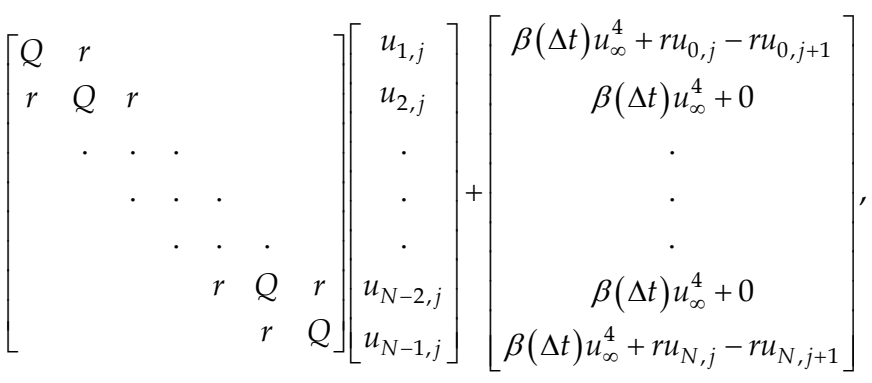

where

$$
M=\left(2+2 r+\beta(\Delta t)\left(u_{i, j}^{2}+u_{\infty}^{2}\right)\left(u_{i, j}+u_{\infty}\right)\right)
$$

and

$$
Q=\left(2-2 r+\beta(\Delta t) u_{\infty}\left(u_{i, j}^{2}+u_{\infty} u_{i, j}+u_{\infty}^{2}\right)\right)
$$

i.e. $\mathbf{A} \mathbf{u}_{j+1}=\mathbf{B} \mathbf{u}_{j}+\mathbf{d}_{j}$, where the matrices $\mathbf{A}$ and $\mathbf{B}$ of order $(N-1)$ are as shown in (4.16), $\mathbf{u}_{j+1}$ denotes the column vector with components $u_{1, j+1}, u_{2, j+1}, \ldots, u_{N-1, j+1}$, and $\mathbf{d}_{j}$ denotes the column vector of known boundary values and zeros. Hence, 


$$
\mathbf{u}_{j+1}=\mathbf{A}^{-\mathbf{1}} \mathbf{B} \mathbf{u}_{j}+\mathbf{A}^{-\mathbf{1}} \mathbf{d}_{j},
$$

that may be expressed more conveniently as

$$
\mathbf{u}_{j+1}=\mathbf{C} \mathbf{u}_{j}+\mathbf{f}_{j}
$$

in which $\mathbf{C}=\mathbf{A}^{-1} \mathbf{B}$ and $\mathbf{f}_{j}=\mathbf{A}^{-1} \mathbf{d}_{j}$.

Theorem 4.1: For the scheme (4.12) norm of the error for $j$ th time step is less than or equal to $\|\mathbf{C}\|^{j}\left\|\mathbf{e}_{0}\right\|$, where $\mathbf{e}_{0}$ is the error of the initial values.

Proof : Applying recursively from (4.20) leads to

$$
\begin{aligned}
\mathbf{u}_{j} & =\mathbf{C} \mathbf{u}_{j-1}+\mathbf{f}_{j-1}=\mathbf{C}\left(\mathbf{C u}_{j-2}+\mathbf{f}_{j-2}\right)+\mathbf{f}_{j-1}= \\
& =\mathbf{C}^{2} \mathbf{u}_{j-2}+\mathbf{C} \mathbf{f}_{j-2}+\mathbf{f}_{j-1}= \\
& =\ldots \\
& =\mathbf{C}^{j} \mathbf{u}_{0}+\mathbf{C}^{j-1} \mathbf{f}_{0}+\mathbf{C}^{j-2} \mathbf{f}_{1}+\ldots+\mathbf{f}_{j-1} .
\end{aligned}
$$

Perturb the vector of initial values $u_{0}$ to $u_{0}^{*}$. The exact solution at the $j$ th time-row will then be

$$
\mathbf{u}_{j}^{*}=\mathbf{C}^{j} \mathbf{u}_{0}^{*}+\mathbf{C}^{j-1} \mathbf{f}_{0}+\mathbf{C}^{j-2} \mathbf{f}_{1}+\ldots+\mathbf{f}_{j-1}
$$

If the perturbation or error vector $\mathbf{e}$ is defined by $\mathbf{e}=\mathbf{u}^{*}-\mathbf{u}$, it follows by Eqs. (4.21) and (4.22) that

$$
\mathbf{e}_{j}=\mathbf{u}_{j}^{*}-\mathbf{u}_{j}=\mathbf{C}^{j}\left(\mathbf{u}_{0}^{*}-\mathbf{u}_{0}\right)=\mathbf{C}^{j} \mathbf{e}_{0}, j=1 \ldots J
$$

Hence, for compatible matrix and vector norms,

$$
\left\|\mathbf{e}_{\mathrm{j}}\right\| \leq\left\|\mathbf{C}^{\mathrm{j}}\right\|\left\|\mathbf{e}_{\mathbf{0}}\right\| \leq\|\mathbf{C}\|^{\mathrm{j}}\left\|\mathbf{e}_{0}\right\| .
$$

Since the necessary and sufficient condition for the difference equations to be stable when the solution for the partial differential equation does not increase as $t$ increases (J. D. Smith, 1985), is $\|C\| \leq 1$, in the following theorem we prove it for the scheme (4.12).

Theorem 4.2: The following three statements for the non-standard implicit scheme (4.12) satisfy

i. $\quad$ Matrix $C$ in Eq. (4.20) is symmetric with real values.

ii. $\quad \mid \mathbf{C} \|<1$

iii. The nonstandard implicit scheme (4.12) is unconditionally stable.

Proof (i) From matrix equation (4.16) it is obvious that matrix $\mathbf{C}$ is a real tridiagonal matrix. Since $\mathbf{A}$ and $\mathbf{B}$ are both symmetric and commute, matrix $\mathbf{C}$ is symmetric with real values, (J. D. Smith, 1985).

Proof (ii) Since matrix $\mathbf{C}$ is real and symmetric, $\|\mathbf{C}\|_{2}=\rho(\mathbf{C})=\max _{s}\left|\mu_{s}\right|$, therefore the scheme (4.12) will be stable when $\|\mathbf{C}\|_{2}=\max _{s}\left|\mu_{s}\right| \leq 1$, where $\mu_{s}$, for $s=1, \ldots, N$, are eigenvalues of the matrix $\mathbf{C}$. On the other hand the eigenvalues of matrix $\mathbf{C}$ are in the following form 


$$
\mu_{s}=\left(\frac{Q+2 r \cos s \pi /(N+1)}{M+2 r \cos s \pi /(N+1)}\right) \quad s=1, \ldots, N .
$$

Thus from (4.17) and (4.18) we have

$$
\|\mathbf{C}\|_{2}=\rho(\mathbf{C})=\max _{s}\left|\frac{Q+2 r \cos s \pi /(N+1)}{M+2 r \cos s \pi /(N+1)}\right|<1 \text { for all } r>0 .
$$
Now using Theorem 4.1, equation (4.24) leads to $\lim _{j \rightarrow \infty}\left\|\mathbf{e}_{j}\right\| \leq \lim _{j \rightarrow \infty}\|\mathbf{C}\|^{j}\left\|\mathbf{e}_{0}\right\|=0$. This proves
statement (iii).

\section{Numerical results}

\subsection{Numerical solutions for Work Example 2}

Explicit and implicit schemes for equations (4.1)-(4.3), and (4.10)-(4.12) are numerically integrated. We computed and plotted the approximate solution to the problem (3.3), for $U_{1}=U_{2}=0$ and various values of $\beta=u_{\infty}=2, \beta=u_{\infty}=6$, and $\beta=u_{\infty}=20$, where $\Delta x=0.02$ and $\Delta t=1 / 5001$. We first chose $\beta=u_{\infty}=2$, figures 5(a) and 6(a) show temperature profiles obtained based on three schemes for explicit models introduced by (P. M. Jordan, 2003; W. Dai \& S. Su, 2004), and three schemes of this work, respectively. It can be seen from figure 6(a) that all of our schemes in figure 6(a) are stable while the scheme (1) in figure 5(a) of Ref. (P. M. Jordan, 2003; W. Dai \& S. Su, 2004) is unstable.

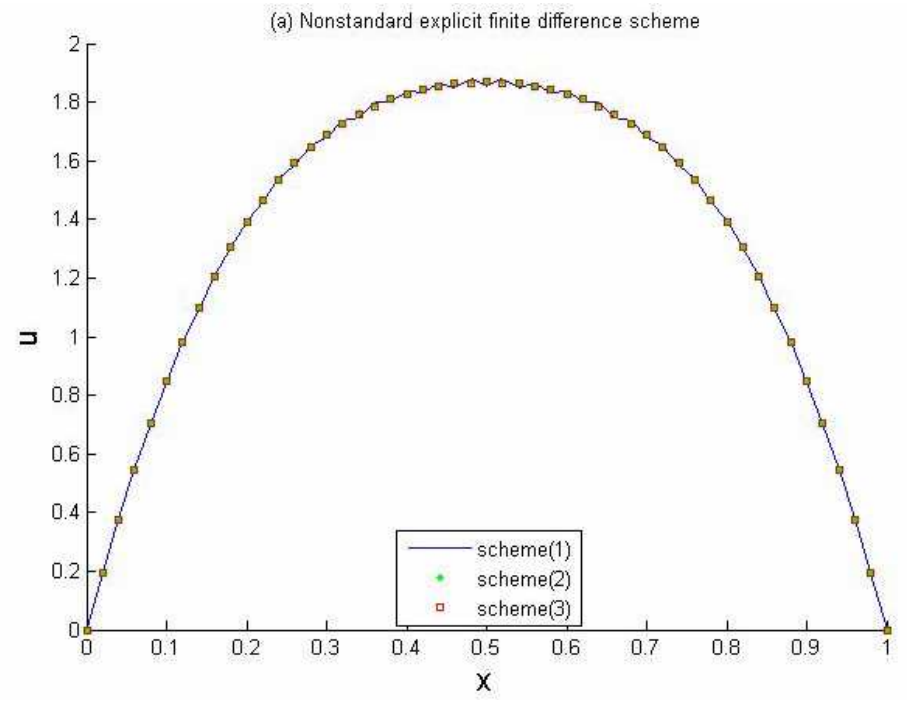

Fig. 5(a). For $\beta=u_{\infty}=2$, scheme (1) There explicit nonstandard finite difference scheme given by Jordan (2003) is plotted in Eq. (4.1) is not stable, while schemes (2) and (3) given in Eqs. (4.2) and (4.3) are stable.

We then chose $\beta=u_{\infty}=6$, and the results were plotted in figures $5(\mathrm{~b}), 5(\mathrm{c})$ and $6(\mathrm{~b})$. The solution obtained based on Eq. (4.1) is not convergent as shown in figure $5(\mathrm{~b})$, while the three implicit schemes of us are stable as shown in figure 6(b). 
Finally, $\beta=u_{\infty}=20$, it can be seen from figures 5(d) and 5(e) and figures 6(c) and 6(d) that neither of the solutions based on Eqs. (4.1), (4.2) and (4.10), (4.11) converge to the correct solution, while the schemes, in Eqs. (4.3) and (4.12) are still stable and convergent.

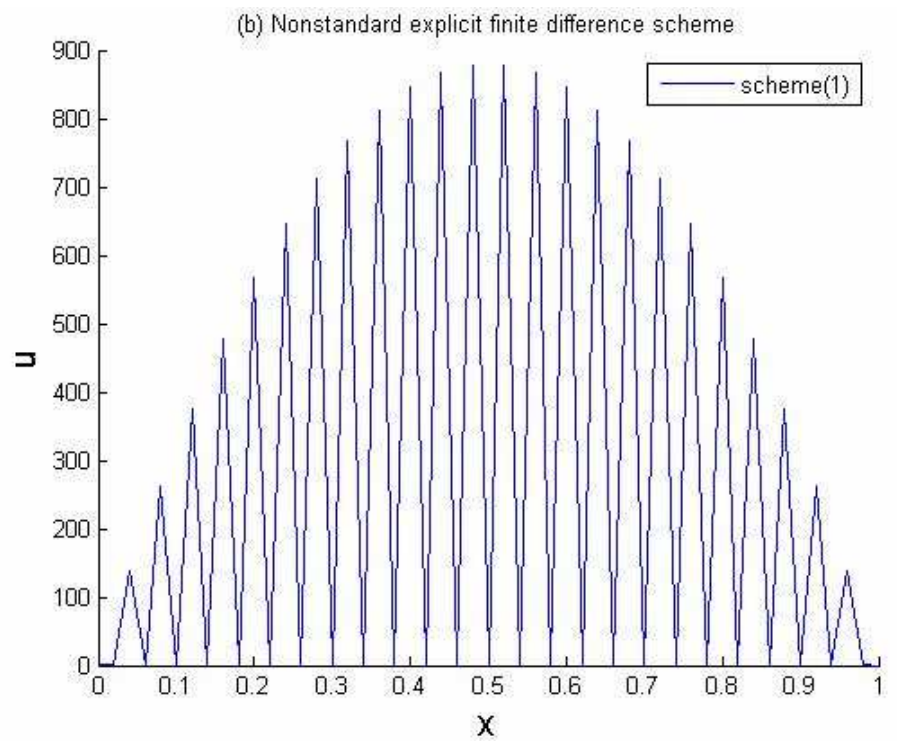

Fig. 5(b). For $\beta=u_{\infty}=6$, scheme (1), given in Eq. (4.1), by Jordan (2003) does not converge.

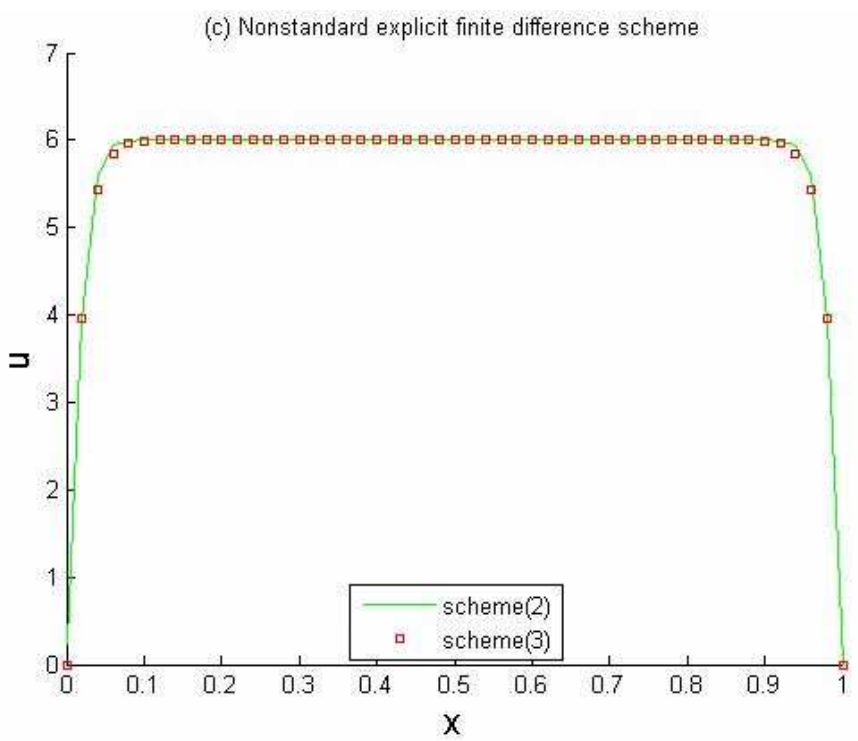

Fig. 5(c). For $\beta=u_{\infty}=6$, schemes (2) and (3), given in Eqs. (4.2) and (4.3), converge to the correct solution. 


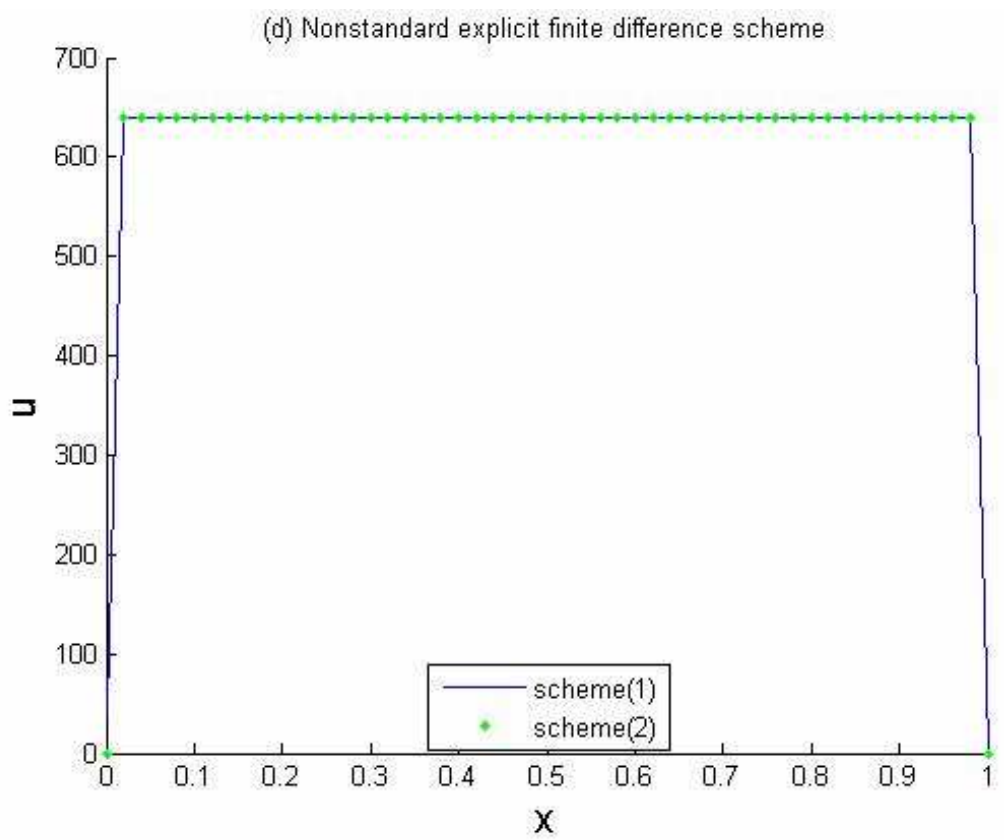

Fig. 5(d). For $\beta=u_{\infty}=20$, schemes (1) and (2), given in Eqs. (4.1) and (4.2), converge but do not converge to the correct solution.

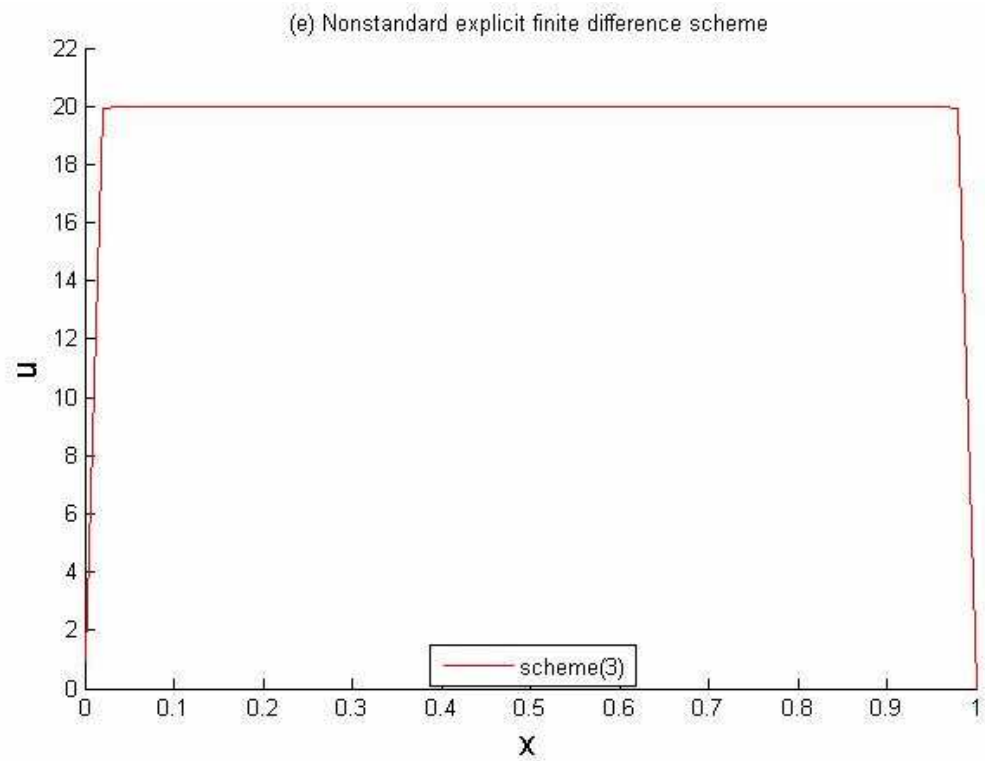

Fig. 5(e). For $\beta=u_{\infty}=20$, scheme (3), given in Eq. (4.3), converge to the correct solution. 


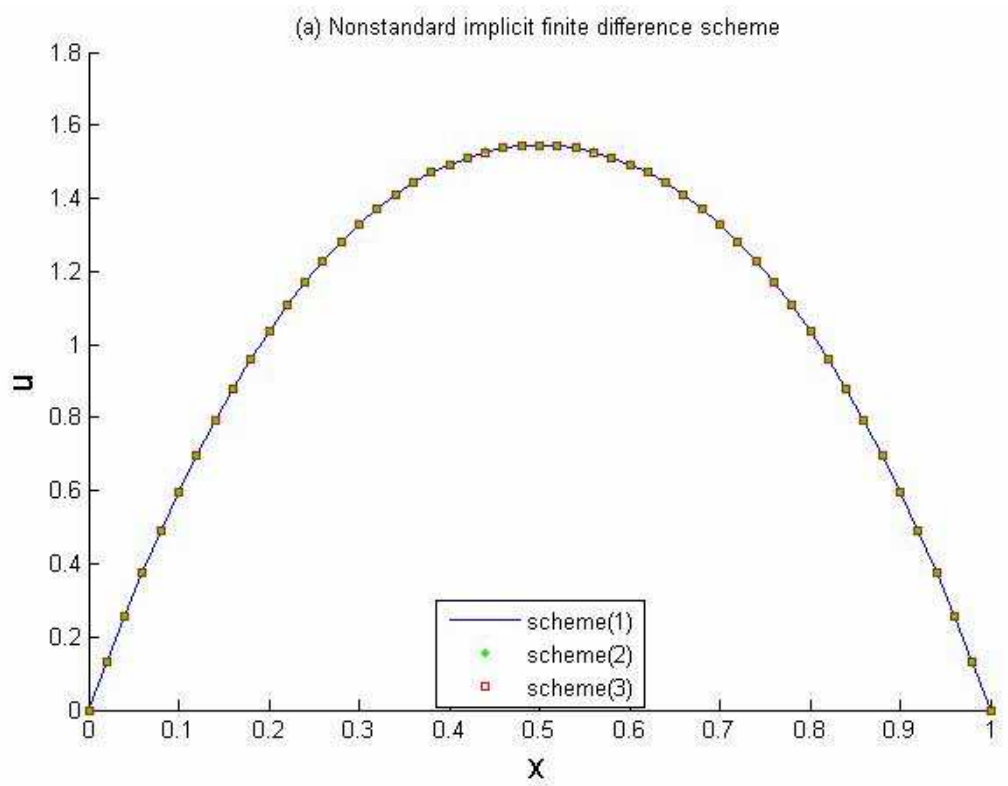

Fig. 6(a). For $\beta=u_{\infty}=2$, three schemes given by Eqs. (4.10), (4.11) and (4.12) converge to the correct solution.

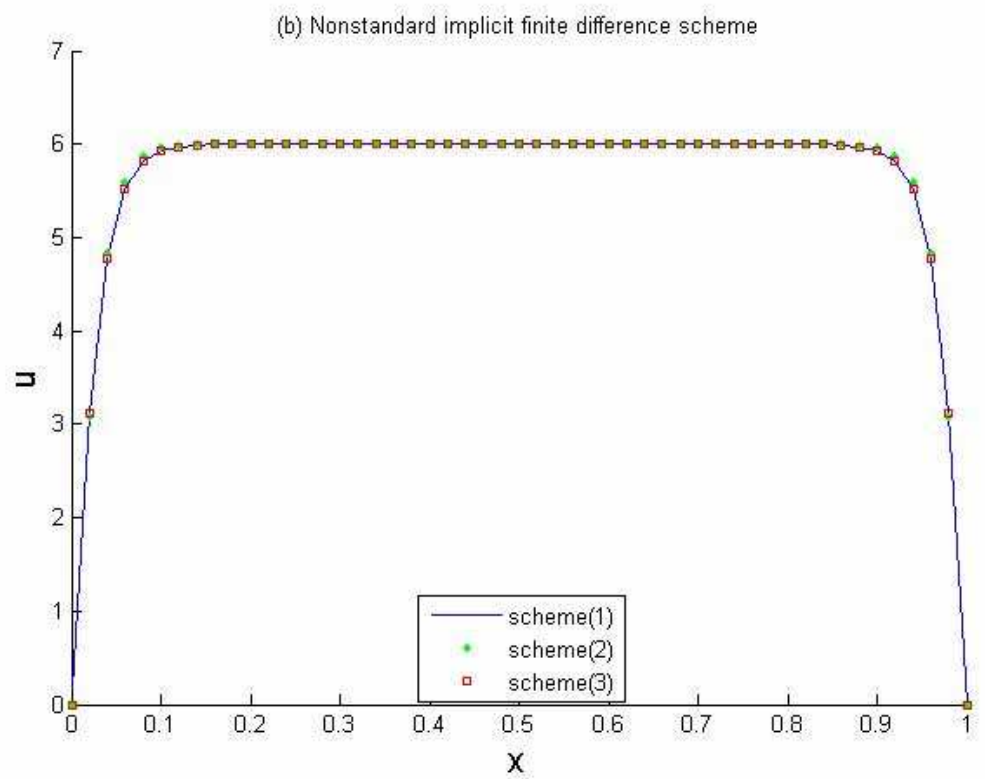

Fig. 6(b). For $\beta=u_{\infty}=6$, schemes (1), (2) and (3) based on Eqs. (4.10), (4.11) and (4.12), for Work Example 2 are shown. All of three implicit schemes are stable. 


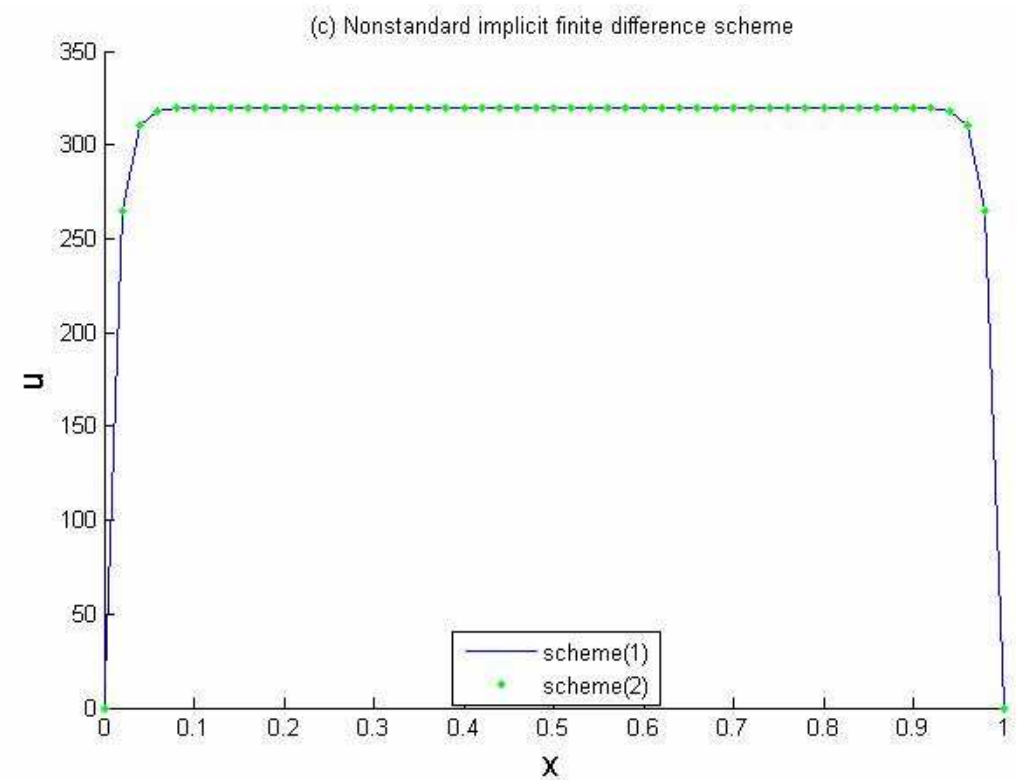

Fig. 6(c). For $\beta=u_{\infty}=20$, schemes (1) and (2), given in Eqs. (4.10) and (4.11), converge but do not converge to the correct solution.

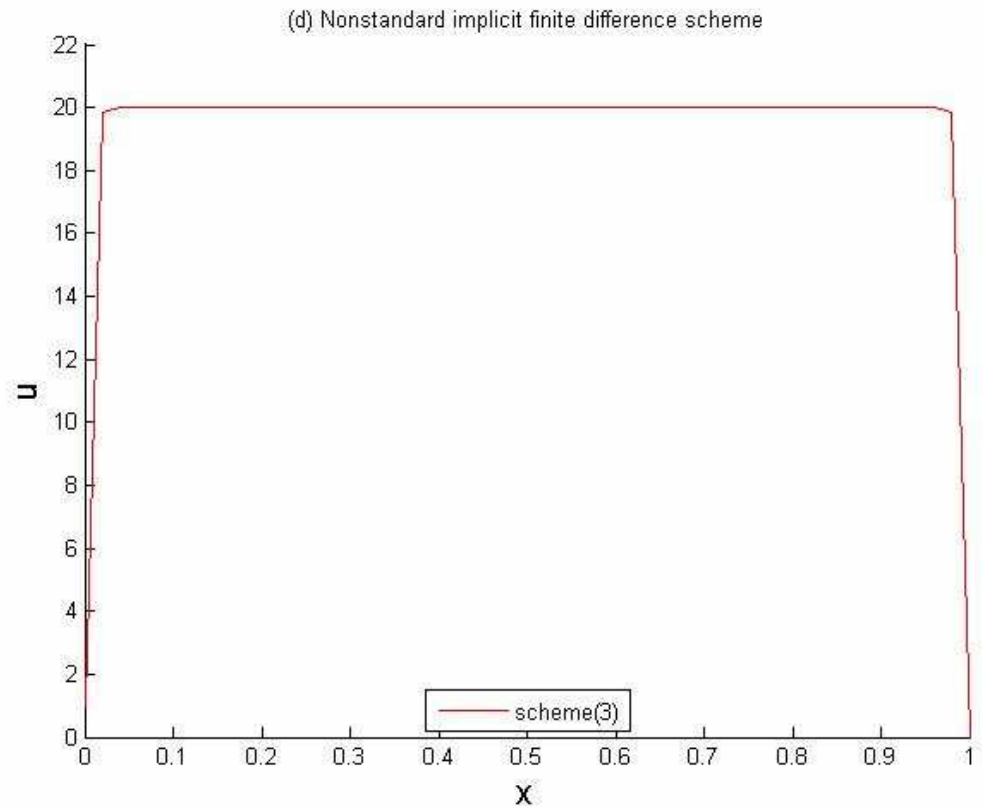

Fig. 6(d). For $\beta=u_{\infty}=20$, scheme (3), given in Eq. (4.12) is stable and converges to the correct solution. 


\subsection{Numerical solutions for Work Example 3}

The approximate solutions to the problem (3.6) are computed and plotted using the finite difference schemes given in Eqs. (4.4)-(4.6) and (4.13)-(4.15) for $t=1, \mathrm{U}_{1}=U_{2}=0$ with $\beta=u_{\infty}=2$, in Work Example 2 and $\beta=u_{\infty}=2, \alpha=4$ in Work Example 3, where $\Delta x=0.02, \Delta t=1 / 5001$.

Figure 7(a) shows the temporal evolution of the temperature profiles corresponding to initial boundary value problem (3.3) and (3.6), for $\beta=u_{\infty}=2$, and $\alpha=4$, where numerical results for explicit schemes are plotted. It can be seen from figure $7(\mathrm{a})$ that the solution of problem without convection term in scheme (1) begun to oscillate, while all of the solution profiles for problem (3.6) are stable.

(a) Nonstandard explicit finite difference schemes with/without convection term

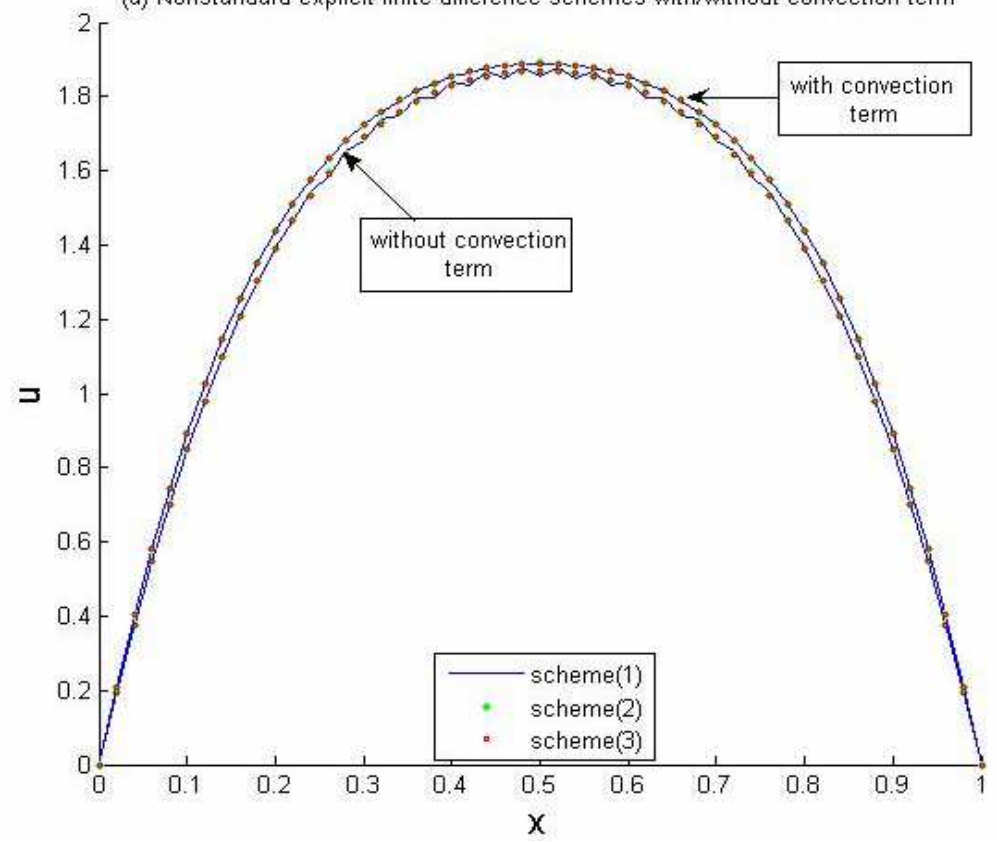

Fig. 7(a). For $\beta=u_{\infty}=2$, plots of explicit schemes (1), (2) and (3) with convection term $(\alpha=4)$ and without convection term are shown.

Figure 7(b) shows the temperature profiles corresponding to initial boundary value problem (3.3) and (3.6), for $\beta=u_{\infty}=2, \alpha=4$ where numerical results for implicit schemes are plotted. All the proposed schemes with/without convection terms are stable when implicit schemes are used.

Numerical results show that solution profile for implicit schemes are unconditionally stable for small values as well as the large values of the equation parameters. The theoretical stability analysis in Section 4.3 for implicit scheme (4.13) supports our numerical conclusions. The theoretical stability analysis for implicit schemes (4.14) and (4.15) may be done in the similar way. The convection term's effect is considered in Figs. 7(a) and 7(b) for explicit and implicit schemes respectively. It is shown that the schemes with convection term reach the steady state sooner. 


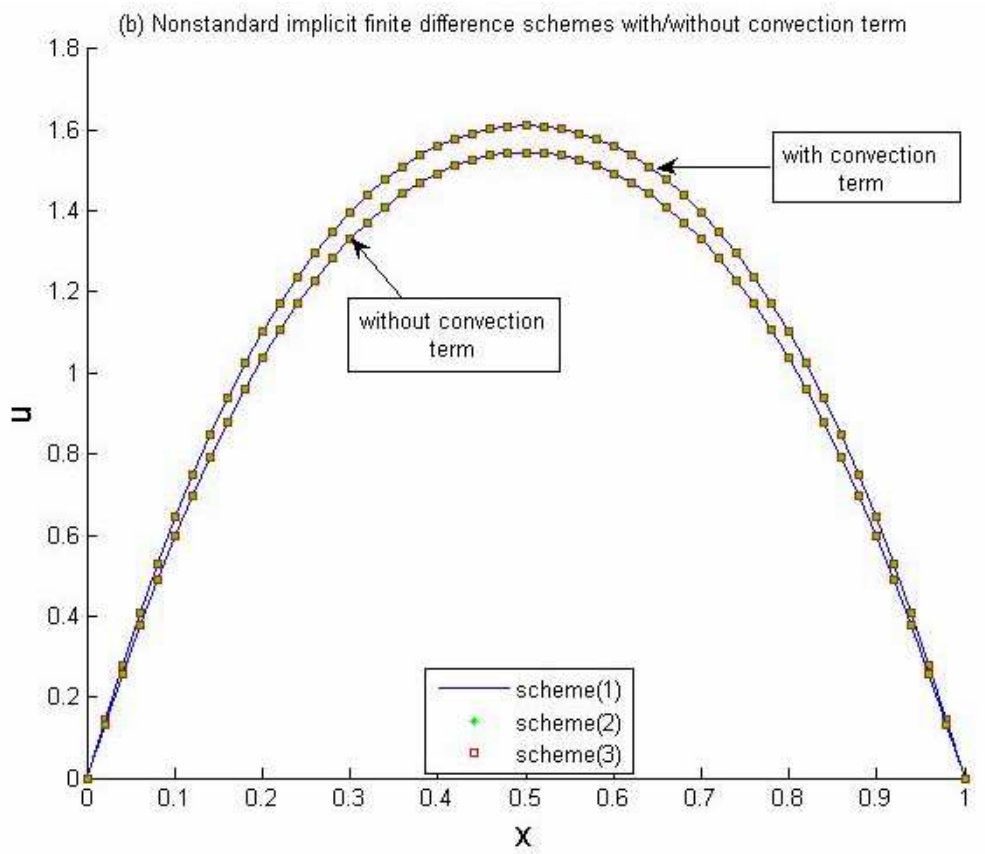

Fig. 7(b). For $\beta=u_{\infty}=2$, implicit schemes (1), (2) and (3) with convection term $(\alpha=4)$ and without convection term is shown.

Our findings suggest that Regulation 4 is a serious property for a general nonstandard finite difference scheme because, otherwise it leads to instability. i.e. either the scheme does not converge or it converges to a wrong solution.

\section{References}

A. Malek, S. H. Momeni-Masuleh, A mixed collocation-finite difference method for 3D microscopic heat transport problems. J. Comput. Appl. Math. 217 (2008), no. 1, 137147.

A. Mohammadi, A. Malek, Stable non-standard implicit finite difference schemes for nonlinear heat transfer in a thin finite rod. J. Difference Equ. Appl. 15 (2009), no. 7, 719728.

D. R. Croft, D. G. Lilly, Heat transfer calculations using finite difference equations. Applied Science Publishers, 1977.

D. Y. Tzou, Macro-To Micro-Scale Heat Transfer: The Lagging Behavior (Chemical and Mechanical Engineering Series). Taylor \& Francis, 1997.

E. Battaner, Astrophysical Fluid Dynamics, Cambridge University Press, Cambridge, 1996.

G. Ben-Yu, Spectral Methods and Their Applications. World Scientific, 1998.

H. K. Versteeg, W. Malalasekera, An Introduction to Computational Fluid Dynamics: The Finite Volume Method. Addison-Wesley. 1996.

H. Heidari, A. Malek, Null boundary controllability for hyperdiffusion equation. Int. J. Appl. Math. 22 (2009), no. 4, 615-626. 
H. Heidari; H. Zwart, A. Malek, Controllability and Stability of 3D Heat Conduction Equation in a Submicroscale Thin Film. Department of Applied Matematics, University of Twente, Netherlands, 2010, 1-21.

H. S. Carslaw and J. C. Jaeger, Conduction of Heat in Solids, 2nd Ed., Oxford University Press, New York, 1959.

J. C. Jaeger, Conduction of heat in a solid with a power law of heat transfer at its surface, Proc. Camb. Phil. Soc., 46 (1950), 634-641.

J. D. Smith, Numerical Solution of Partial Differential Equation, Clarendon Press, Oxford, 1985.

J. M. Bergheau, R. Fortunier, Finite Element Simulation of Heat Transfer. ISTE Ltd, 2010.

L. C. Burmeister, Convective Heat Transfer, 2nd Ed., Wiley, New York, 1993.

M. N. Ozisik, Boundary Value Problems of Heat Conduction, Dover, New York, 1989.

M. Necati Ozisik, M. Necati Czisik, Necati Ozisik. Finite Difference Methods in Heat Transfer, Crc Press, 1994.

O. P. Le Maitre, O. M. Knio, Spectral Methods for Uncertainty Quantification: With Applications to Computational Fluid Dynamics. Springer, 2010.

P. M. Jordan, A nonstandard finite difference scheme for a nonlinear heat transfer in a thin finite rod, J. Diff. Eqs. Appl., 9 (2003), 1015-1021.

R. E. Mickens, Nonstandard finite difference schemes for differential equations, J. Diff. Eqs. Appl., 8 (2002), 823-857.

R. E. Mickens, Nonstandard Finite Difference Models of Differential Equations. World Scientific, Singapore, 1994.

R. E. Mickens, Nonstandard finite difference schemes for reaction-diffusion equations, Numer Methods Partial Diff. Eqs. 15(1999), 201-214.

R. E. Mickens, and A.B Gumel, Construction and analysis of a nonstandard finite difference scheme for the Burgers-Fisher equation. J. Sound Vib. 257 (2002), 791-797.

R. E. Mickens, Advances in the applications of nonstandard finite difference schemes. World Scientific, London, 2005.

R. Siegel and J. H. Howell, Thermal Radiation Heat Transfer, McGraw-Hill, New York, 1972.

R. W. Lewis, P. Nithiarasu, K. N. Seetharamu, Fundamentals of the Finite Element Method for Heat and Fluid Flow, John Wiley \& Sons Ltd, 2005.

S. H. Momeni-Masuleh, A. Malek, Hybrid pseudospectral-finite difference method for solving a 3D heat conduction equation in a submicroscale thin film. Numer. Methods Partial Differential Equations 23 (2007), no. 5, 1139-1148.

S. H. Momeni-Masuleh, A. Malek, Pseudospectral Methods for Thermodynamics of Thin Films at Nanoscale. African Physical Review, 2007, 35-36.

S. V. Patankar, Numerical Heat Transfer and Fluid Flow (Hemisphere Series on Computational Methods in Mechanics and Thermal Science). T \& F / Routledge, 1980.

W. Dai and S. Su, "A nonstandard finite difference scheme for solving one dimensional nonlinear heat transfer," Journal of Difference Equations and Applications 10 (2004), 1025-1032. 


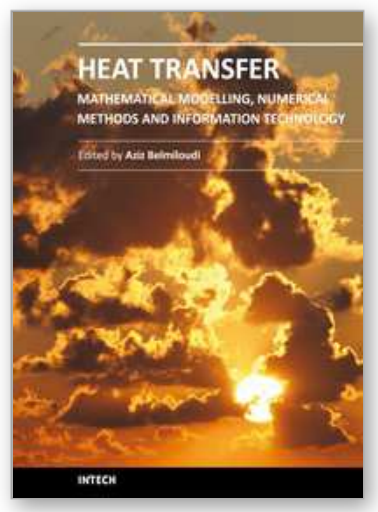

\section{Heat Transfer - Mathematical Modelling, Numerical Methods and Information Technology}

Edited by Prof. Aziz Belmiloudi

ISBN 978-953-307-550-1

Hard cover, 642 pages

Publisher InTech

Published online 14, February, 2011

Published in print edition February, 2011

Over the past few decades there has been a prolific increase in research and development in area of heat transfer, heat exchangers and their associated technologies. This book is a collection of current research in the above mentioned areas and describes modelling, numerical methods, simulation and information technology with modern ideas and methods to analyse and enhance heat transfer for single and multiphase systems. The topics considered include various basic concepts of heat transfer, the fundamental modes of heat transfer (namely conduction, convection and radiation), thermophysical properties, computational methodologies, control, stabilization and optimization problems, condensation, boiling and freezing, with many real-world problems and important modern applications. The book is divided in four sections : "Inverse, Stabilization and Optimization Problems", "Numerical Methods and Calculations", "Heat Transfer in Mini/Micro Systems", "Energy Transfer and Solid Materials", and each section discusses various issues, methods and applications in accordance with the subjects. The combination of fundamental approach with many important practical applications of current interest will make this book of interest to researchers, scientists, engineers and graduate students in many disciplines, who make use of mathematical modelling, inverse problems, implementation of recently developed numerical methods in this multidisciplinary field as well as to experimental and theoretical researchers in the field of heat and mass transfer.

\section{How to reference}

In order to correctly reference this scholarly work, feel free to copy and paste the following:

Alaeddin Malek (2011). Applications of Nonstandard Finite Difference Methods to Nonlinear Heat Transfer Problems, Heat Transfer - Mathematical Modelling, Numerical Methods and Information Technology, Prof. Aziz Belmiloudi (Ed.), ISBN: 978-953-307-550-1, InTech, Available from: http://www.intechopen.com/books/heattransfer-mathematical-modelling-numerical-methods-and-information-technology/applications-of-nonstandardfinite-difference-methods-to-nonlinear-heat-transfer-problems

\section{INTECH}

open science | open minds

\section{InTech Europe}

University Campus STeP Ri

Slavka Krautzeka 83/A

51000 Rijeka, Croatia

Phone: +385 (51) 770447

\section{InTech China}

Unit 405, Office Block, Hotel Equatorial Shanghai

No.65, Yan An Road (West), Shanghai, 200040, China

中国上海市延安西路65号上海国际贵都大饭店办公楼 405 单元

Phone: +86-21-62489820 
Fax: +385 (51) 686166

Fax: +86-21-62489821

www.intechopen.com 
(C) 2011 The Author(s). Licensee IntechOpen. This chapter is distributed under the terms of the Creative Commons Attribution-NonCommercialShareAlike-3.0 License, which permits use, distribution and reproduction for non-commercial purposes, provided the original is properly cited and derivative works building on this content are distributed under the same license. 